\title{
Unexpected etiology of dysphagia in an immunocompetent adult
}

\begin{abstract}
Retropharyngeal abscesses are exceptional in adults. The most common are post-traumatic or foreign-body pyogenic abscesses. Tuberculosis remains a rare and unusual cause of these abscesses, particularly reported if underlying cervical vertebra involvement. Exceptionally, they may be the revealing feature of cervical Pott's disease. They are particularly observed in immunocompromised individuals, whereas they remain exceptional in immunocompetent adults. These abscesses remain an exceptional and unusual cause of dysphagia even in tuberculosis endemic countries, and only a few sporadic cases have been reported. We report an original case of isolated dysphagia revealing a tuberculous retropharyngeal abscess associated with very destructive cervical spondylodiscitis, without any neurological signs, occurring in immunocompetent adult woman.
\end{abstract}

Keywords: dysphagia, tuberculous retropharyngeal abscess, immunocompetent adult, tuberculosis, pott's disease
Volume 10 Issue 5 - 2019

\author{
Salem Bouomrani',2, Rym Mesfar ${ }^{1,2}$, \\ Fatma Rekik ${ }^{1,2}$, Samira M'hamdi ${ }^{3,4}$, Salsabil \\ Dabboussi ${ }^{3,4}$ \\ 'Department of Internal medicine, Military Hospital of Gabes, \\ Tunisia \\ ${ }^{2}$ Sfax Faculty of Medicine, University of Sfax, Tunisia \\ ${ }^{3}$ Department of Pneumology, Military Hospital of Tunis, Tunisia \\ ${ }^{4}$ Tunis Faculty of Medicine, Tunis El Manar University, Tunisia
}

\section{Correspondence: Dr Salem Bouomrani, Department of Internal medicine Military Hospital of Gabes, Gabes 6000 , Tunisia, Email salembouomrani@yahoo.fr}

Received:September 14, 2019 | Published:September 25 2019

\section{Introduction}

Retropharyngeal abscesses are exceptional in adults. ${ }^{1}$ The most common are post-traumatic or foreign-body pyogenic abscesses. ${ }^{1,2}$ Their diagnosis is a real emergency because of the risk of rupture into the upper respiratory tract that can be fatal. ${ }^{2-4}$ Tuberculosis remains a rare and unusual cause of these retropharyngeal abscesses, ${ }^{5-7}$ particularly reported if underlying cervical vertebra involvement. ${ }^{1,2}$ More rarely, retropharyngeal abscess may be the revealing feature of cervical tuberculous spondylodiscitis. ${ }^{1,2,8}$ It is particularly observed in immunocompromised individuals, whereas it remains exceptional in immunocompetent adults. ${ }^{1,7}$ We report an original case of isolated dysphagia revealing a tuberculous retropharyngeal abscess associated with very destructive cervical spondylodiscitis, without any neurological signs, occurring in immunocompetent adult woman.

\section{Case report}

A 34-year-old Tunisian woman with no pathological medical history was hospitalized in our department for exploration of isolated upper dysphagia. This dysphagia was incidious, progressively worsening since a month ago, noted for the solids at first and then for solids and liquids. No cervical trauma, toxic substance abuse, accidental intoxication, fever, dyspnoea, dysphonia, or weight loss have been reported. Somatic examination indicated a stiffness of the cervical spine, with in particular a significant and painful limitation of lateral inclinations and bilateral inflammatory cervical lymphadenopathy. The remainder of the somatic examination was without abnormalities, in particular there was no evidence of neurological deficit, visceromegaly, or palpable masses.

Biology showed a marked inflammatory biological syndrome: erythrocyte sedimentation rate at $130 \mathrm{~mm} / \mathrm{H} 1$, a C-reactive protein at $38 \mathrm{mg} / \mathrm{l}$, and a normochromic normocytic anemia at $10 \mathrm{~g} / \mathrm{dl}$ of hemoglobin. Leukocytes, platelets, blood glucose, serum calcium, creatinine, ionogram, transaminases, muscle enzymes, and lipid parameters were within normal limits. X-rays of the cervical spine showed significant damage of the fifth and sixth cervical vertebrae (C5 and C6) with destruction of the vertebral bodies, narrowing of the joint space and marked thickening of the retropharyngeal space. Cervical computed tomography (CT) confirmed the diagnosis of advanced C5-C6 spondylodiscitis (Figures $1 \& 2$ ) and demonstrated a large retropharyngeal abscess exerting a mass effect on aerodigestive structures (Figure 3).

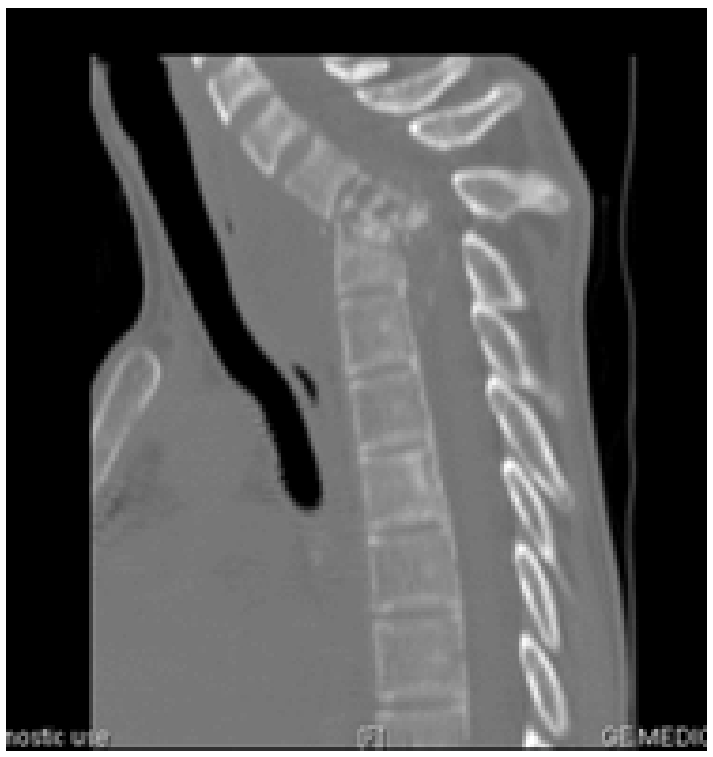

Figure I Sagittal cervical CT/bone window: C5-C6 spondylodiscitis with retropharyngeal abscess. 


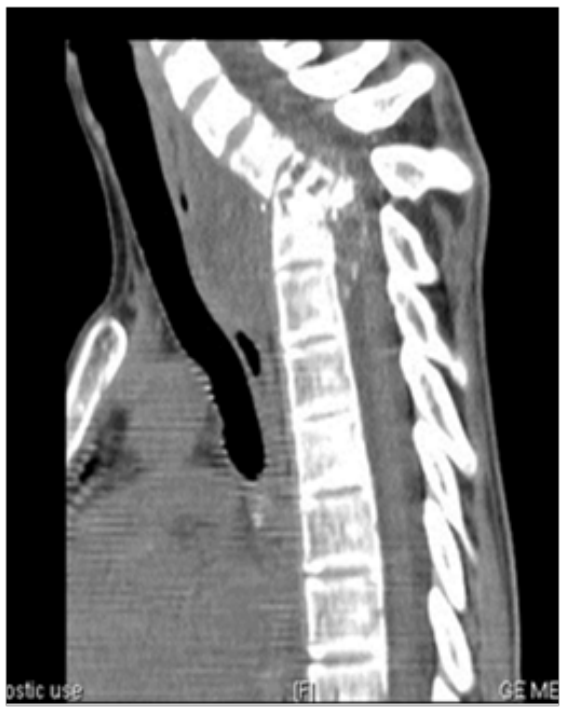

Figure 2 Sagittal cervical CT/parenchymal window: C5-C6 spondylodiscitis with retropharyngeal abscess compressing the aero-digestive structures.

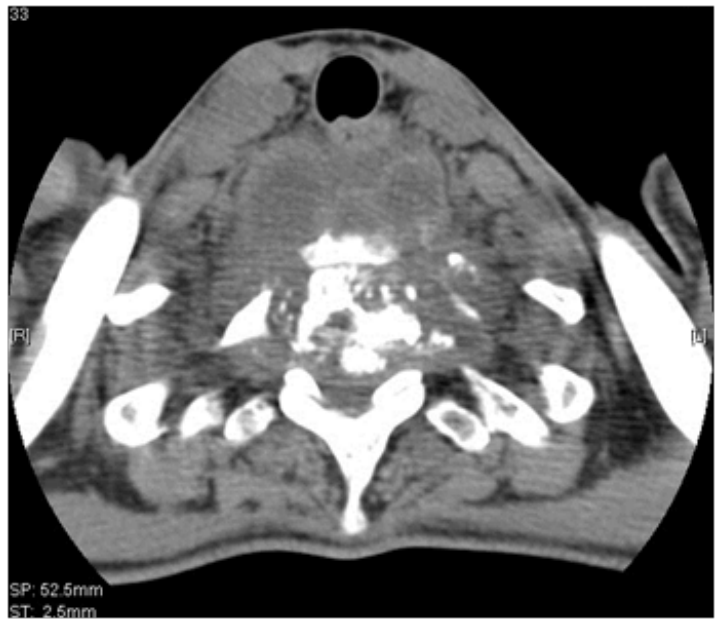

Figure 3 Axial cervical CT/parenchymal window without injection: significant destruction of the vertebral body of C5 with retropharyngeal abscess compressing the aero-digestive structures.

The infectious investigation was negative. Tuberculin skin test was positive at $15 \mathrm{~mm}$. The patient underwent an intraoral aspiration of the retropharyngeal abscess. Microbiology and cytology examination of pharyngeal aspiration showed caseous pus with acid-fast bacilli.

Subsequent investigations failed to identify other active tuberculosis sites: chest X-ray, abdominal ultrasound, and thoracoabdominopelvic CT. Magnetic resonance imaging (MRI) of the cervical spine did not indicate neurological damage. Thus, the diagnosis of cervical tuberculous spondylodiscitis complicated by retropharyngeal cold abscess was retained.

The patient was treated with anti-tuberculosis quadruple therapy combining isoniazid, rifampicin, pyrazinamide and ethambutol for two months with a rapidly favorable course. The dysphagia disappeared after two weeks, the biological parameters returned to normal at the end of the first month, and the two-month CT scan showed the complete disappearance of the retropharyngeal collection.
A dual therapy combining isoniazid and rifampicin was continued for another eight months.

\section{Discussion}

The cervical spine is an extremely rare localization of vertebral tuberculosis. ${ }^{6}$ Its frequency is estimated at $2-5 \%$ of all tuberculous spondylodiscitis. ${ }^{1,9}$ In rare cases, these spondylodiscitis may be complicated by cold para- or retrpharyngeal abscesses ${ }^{2}$ and may be the cause of oropharyngeal obstruction. ${ }^{1,4}$ More rarely, these retropharyngeal abscesses may be associated with sternum ${ }^{10}$ or active pulmonary tuberculosis., ${ }^{71}$ Exceptionally retropharyngeal tuberculous abscess may be primitive, without any cervical spine tuberculosis. ${ }^{3,7,11,12}$ The clinical presentation of these abscesses varies from totally asymptomatic forms ${ }^{12}$ to forms resulting in oropharyngeal obstruction symptoms: odynophagia, dysphagia, dyspnoea, laterocervical swelling, and hoarseness of the voice. , $, 2,4,10^{-}$ $\mathrm{CT}$ and MRI are very useful for the positive diagnosis, the assessment of lesions, and the post-therapeutic follow-up of these abscesses..$^{1,2,8}$ Even in tuberculosis endemic countries, tuberculous retropharyngeal abscess remains an exceptional and unusual cause of dysphagia, ${ }^{10,13,14}$ and only a few sporadic cases have been reported in the global medical literature.

The prognosis is usually favorable under anti-tuberculosis drugs. ${ }^{1,2}$ Surgical drainage is indicated for large abscesses, whereas neurosurgical treatment is only necessary in cases of neurological deficit or vertebral instability. ${ }^{1}$ Early diagnosis and timely and appropriate management are highly recommended because these abscesses can be fatal. ${ }^{4}$ Our observation is distinguished by dysphagia as an inaugural and isolated symptom, the immunocompetent status of the patient, and the absence of neurological signs despite advanced cervical vertebra destruction.

\section{Conclusion}

Tuberculous retropharyngeal abscess remain exceptional in immunocompetent adult subjects. They represent real diagnostic and therapeutic emergencies because of the potential risk of rupture into the upper respiratory tract that can be fatal. This unusual etiology of dysphagia is worthy of mention in front of any dysphagia that not prove itself, especially in countries endemic for tuberculosis.

\section{Acknowledgment}

None.

\section{Conflicts of interest}

Author declares their are no conflits of interest.

\section{References}

1. Benhammou A, Bencheikh R, Benbouzid MA, et al. Cervical Pott's disease revealed by retropharyngeal abscesses. Rev Stomatol Chir Maxillofac. 2007;108(6):543-546.

2. Kooli H, Marreckchi M, Tiss M, et al. Cold parapharyngeal abscess in spondylodiscitis. Presse Med. 2001;30(1):19-21.

3. Singh J, Velankar H, Shinde D, et al. Retropharyngeal cold abscess without Pott's spine. S Afr J Surg. 2012;50(4):137-139.

4. Hugar BS, Chandra YP, Babu PR, et al. Fatal case of retropharyngeal abscess associated with Pott's disease. J Forensic Leg Med. 2013;20(6):567-569. 
5. Alawad AA, Khalifa AF. A Rare Cause of Retropharyngeal Abscess: Cervical Pott's Disease. Am J Trop Med Hyg. 2015;92(5):884.

6. Sierra-Bergua B, Sanchez-Marteles M, Morales-Rull JL, et al. Cervical pain and debility: Tuberculosis of an unusual location. Rev Clin Esp. 2010;210(8):e33-e35.

7. Ekka M, Sinha S. Retropharyngeal abscess as a rare presentation of pulmonary tuberculosis. Lung India. 2015;32(3):262-264.

8. Diom ES, Ndiaye C, Djafarou AB et al. A case of cervical Pott's disease revealed by parapharyngeal abscess. Eur Ann Otorhinolaryngol Head Neck Dis. 2011;128(3):151-153.

9. Lukhele M. Tuberculosis of the cervical spine. $S$ Afr Med J. 1996;86(5):553-556.
10. Al Soub H. Retropharyngeal abscess associated with tuberculosis of the cervical spine. Tuber Lung Dis. 1996;77(6):563-565.

11. Patil C, Patil RK, Deshmukh P, et al. Tuberculous retropharyngeal abscess without cervical spine TB. Asian Pac J Trop Med. 2011;4(3):251-252.

12. Oktem F, Güvenç MG, Yilmaz S, et al. Asymptomatic retropharyngeal abscess related to cervical Pott's disease. Am J Otolaryngol. 2006;27(4):278-280.

13. Christoforidou A, Metallidis S, Kollaras P, et al. Tuberculous retropharyngeal abscess as a cause of oropharyngeal dysphagia. Am J Otolaryngol. 2012;33(2):272-274.

14. Menon E, Baruah P. Tuberculous retropharyngeal abscess revisited. BMJ Case Rep. 2014;2014. pii: bcr2013202514. 